\title{
Estimation and Modeling of Fluctuating Wind Amplitude and Phase Spectrum Using APES Algorithm Based on Field Monitored Data
}

\author{
Dan-hui Dan $\mathbb{D D}^{1}{ }^{1}$ Xiang-jie Wang, ${ }^{1}$ Xing-fei Yan, ${ }^{2}$ and Wei Cheng ${ }^{1}$ \\ ${ }^{1}$ Department of Bridge Engineering, Tongji University, Shanghai 200092, China \\ ${ }^{2}$ Shanghai Urban Construction Design Research Institute, Shanghai 200125, China
}

Correspondence should be addressed to Dan-hui Dan; dandanhui@tongji.edu.cn

Received 19 July 2017; Accepted 7 March 2018; Published 11 April 2018

Academic Editor: Marc Thomas

Copyright (C) 2018 Dan-hui Dan et al. This is an open access article distributed under the Creative Commons Attribution License, which permits unrestricted use, distribution, and reproduction in any medium, provided the original work is properly cited.

\begin{abstract}
The fluctuating wind power spectrum (FWPS) in given specifications could only represent the second-order probabilistic characteristic, which indicates that it is not capable of fully expressing the stochastic wind field. Estimation and modeling of the fluctuating wind amplitude spectrum (FWAS) as well as the fluctuating wind phase spectrum (FWPhS) by using measured wind velocity data can make up for the deficiencies mentioned above. A high-resolution nonparametric spectral estimation algorithm — amplitude and phase estimation (APES) — is used to estimate the FWAS and the FWPhS, using the field measured wind velocity data of a certain cable-stayed bridge in Shanghai, China. An empirical expression (eFWAS) is introduced by dimensional analysis to model the random FWAS, and its specific Davenport form is proposed according to field measured data. The parameters of the Davenport eFWAS model are estimated by using the above measured FWAS, and three specific applications of this model are put forward when different known conditions are met. Compared with the measured FWAS, the stochastic Davenport eFWAS model proposed in this paper can accurately describe the statistical properties of the local wind field and improve the modeling accuracy of the FWAS, which is important in antiwind structural design and safety assessment.
\end{abstract}

\section{Introduction}

The vibration induced by wind load may pose a serious threat to the safety of the structure for super-high or longspan engineering structures; thus, wind load has become one of the factors that is considered in the design of these structures [1]. Monitoring, analysis, and modeling of the wind load, as well as analyzing the wind effects, becomes not only one of the challenges in the preplanning and design, but also an important issue for the safety evaluation of the completed structures [2]. In geophysical meteorological conditions, the wind load energy is mostly concentrated in lowfrequency regions [3]. Accurately understanding the wind load information, especially in low-frequency regions, is of great importance in the antiwind design of structures. With respect to wind-induced response of structures, the wind load in low-frequency regions can be divided into two parts: load due to mean wind and load due to fluctuating wind. It is generally believed that the former is the energy component in low-frequency region that affects the static behavior of structures, while the latter component is in a slightly higher frequency region and is the main factor responsible for wind-induced vibrations. The measurement, analysis, and modeling of wind loads are mainly conducted for the fluctuating wind, which is characterized by turbulence intensity, turbulence integral scale, wind spectrum, and so on [4]. For the neutral layer wind, fluctuating wind is composed of many vortexes of different wind speeds and can be expressed as a zero-mean ergodic stationary random process [5]. Thus, the wind spectrum has become an important parameter for describing the energy distribution in the frequency domain.

There are two types of fluctuating wind velocity spectra: the power spectrum and the amplitude spectrum. There is 
a long study history of the former, which can be traced back to the Kármán and Theodore spectrum [6] in 1948. This spectrum describes the horizontal fluctuating wind power spectrum (FWPS) that is independent of height. On this basis, many FWPSs have been put forward such as the Panofsky spectrum (1959), Davenport Spectrum (1961), Kaimal spectrum (1972), and the Simiu spectrum (1974) [7]. The FWPSs mentioned above are based on the Kolmogorov hypothesis, which considers that wind is isotropic and located in the neutral layer. After several decades of continuous improvements, some of these have been adopted by the national engineering design specifications, thereby creating the basis for antiwind designs. However, owing to the influence of the geographical and meteorological conditions of the wind field, the actual wind spectrum is different from the spectra derived from specifications; therefore, field measurements are important supplements to wind spectrum derived from specifications [8].

On the other hand, these existing wind power spectra describe the characteristics of turbulent wind from energy distribution in the frequency domain, and it only reflects the second-order characteristics of the fluctuating wind. Therefore, other richer probabilistic information is lost, including information about the phase, without which it is difficult to completely reestablish the random nature of the fluctuating wind. As a result, spectral representation method $[9,10]$ and linear regression filter method [11] are adopted when generating simulated wind time history from the FWPSs, which yields a large computation. If the wind time history can be derived through amplitude spectrum and phase spectrum, the complete statistical properties can be conserved, while the amount of simulation computation is also reduced. Li and Zhang [12] obtained an empirical formula by Fourier transform using measured data and demonstrated the advantage of using the fluctuating wind amplitude spectrum (FWAS) to express the statistical properties of fluctuating wind. However, the poor frequency resolution and large estimation error of the amplitude and phase result in an FWAS with inaccurate computation results while using Fourier transform.

In recent years, owing to the development of structural health monitoring technology, wind speed and direction monitoring system has been installed in many engineering structures. By using the field measured wind speed data recorded by the system, an FWAS model that reflects the statistical characteristics of the wind field is proposed. It can realize the safety monitoring of the structure under wind load and can also provide reference for other projects. In this paper, taking advantage of the good results from the amplitude and phase estimation using the amplitude and phase estimation algorithm (APES) [13-15], the FWAS and fluctuating wind phase spectrum (FWPhS) were estimated using wind data of Shanghai Minpu Second Bridge Health Monitoring System. Their statistical properties were also studied. First, a more generalized expression of the FWAS is derived according to dimensional analysis. Next, the parameters are obtained using measured fluctuating wind amplitude spectra. Then, an FWAS model containing the stochastic properties is derived.

\section{Estimation of FWAS and FWPhS Based on APES}

2.1. Signal Model of Fluctuating Wind. As mentioned above, the measured turbulence wind velocity could be regarded as a stationary Gaussian process under stationary wind conditions and is the superposition of many harmonic waves and stationary noise,

$$
u(t)=\sum_{r=1}^{M} U\left(\omega_{r}\right) \sin \left(\omega_{r} t+\varphi\left(\omega_{r}\right)\right)+w_{\omega_{r}}(t)
$$

where $u(t)$ is a horizontal fluctuating wind velocity, $\omega_{r}$ is the frequency of the $r_{\text {th }}$ resonant component, $U\left(\omega_{r}\right)$ and $\varphi\left(\omega_{r}\right)$ are its amplitude and phase, respectively, and $w_{\omega_{r}}(t)$ is the noise of this frequency.

At any frequency of interest, the wind speed $u(t)$ can be written as a sum of the sine term modulated by the amplitude function $U(\omega)$ and a residual noise term $w_{\omega}(t)$ :

$$
u(t)=U(\omega) \sin (\omega t+\varphi(\omega))+w_{\omega}(t)
$$

where $U(\omega)$ and $\varphi(\omega)$ are, respectively, the amplitude spectrum function and phase spectrum function at the frequency $\omega, w_{\omega}(t)$ contains all the residual sine terms except at the frequency $\omega$ and a random noise component $w(n)$.

\subsection{Estimation of FWAS and FWPhS. APES algorithm is a} high-resolution nonparametric estimation method for amplitude and phase spectrum, which was first proposed by Li and Stoica in 1996. Just like the Capon algorithm (Capon, 1983) [16], this type of nonparametric spectral estimation method is mostly used for complex signal and nondamped signal [15]. Stoica et al. [17] realized APES and Capon for real signal at the same time. Owing to the fact that the fluctuating wind signal is the real signal, the improved real signal APES spectrum estimation is used to estimate the spectra of wind speed signal as follows.

Equation (2) can be written as

$$
u(t)=U_{I}(\omega) \cos (\omega t)+U_{Q}(\omega) \sin (\omega t)+w_{\omega}(t),
$$

where $U_{I}(\omega)=U(\omega) \cos (\varphi(\omega))$ and $U_{Q}(\omega)=U(\omega) \sin (\varphi(\omega))$.

Let the wind velocity data $u(t)$ obtained through continuous monitoring be expressed as $N$ observation data $u(n)$, $n=0,1, \ldots, N-1$. Then each observation data point can be written as

$$
u(n)=U_{I}(\omega) \cos (\omega n)+U_{\mathrm{Q}}(\omega) \sin (\omega n)+w_{\omega}(n) .
$$

Define an $M \times L$-order Hankel matrix of the observation data as follows:

$$
\mathbf{Y}=\left[\mathbf{y}_{l}\right]
$$

where $\mathbf{y}_{l}=\{u(l), u(l+1), \ldots, u(l+M-1)\}^{T}, l=0,1, \ldots, L-1$; $M$ is a user-defined parameter also known as finite impulse response (FIR) filter length, $M \leq N / 2$; and $L=N-M+1$ represents the total number of snapshot times for the time series. 
Let

$$
\begin{aligned}
\boldsymbol{\Theta}(\omega) & =\left[\begin{array}{ll}
U_{I}(\omega) & U_{\mathrm{Q}}(\omega) \\
U_{\mathrm{Q}}(\omega) & -U_{I}(\omega)
\end{array}\right], \\
\boldsymbol{\theta}(\omega) & =\left[\begin{array}{ll}
U_{I}(\omega) & U_{\mathrm{Q}}(\omega)
\end{array}\right]^{T}=\boldsymbol{\Theta} \cdot\left[\begin{array}{ll}
1 & 0
\end{array}\right]^{T}, \\
\mathbf{V}_{\omega}(l) & =\left[\begin{array}{ll}
\cos \omega l & \sin \omega l]
\end{array}\right] \\
\mathbf{A}_{M}(\omega) & =\left[\begin{array}{cc}
1 & \cos \omega \\
\cos \omega \\
\vdots & \vdots \\
\cos \omega(M-2) & \sin \omega(M-2) \\
\cos \omega(M-1) & \sin \omega(M-1)
\end{array}\right], \\
\mathbf{B}_{\omega}(l) & =\left[\begin{array}{ll}
\cos \omega l & \sin \omega l \\
\sin \omega l & \cos \omega l
\end{array}\right] .
\end{aligned}
$$

Then the signal model can be written as

$$
\begin{aligned}
& \mathbf{y}_{l}=\mathbf{A}_{M}(\omega) \cdot \mathbf{B}_{\omega}(l) \cdot \boldsymbol{\theta}(\omega)+\mathbf{n}_{l}, \\
& \mathbf{y}_{l}=\mathbf{A}_{M}(\omega) \cdot \boldsymbol{\Theta}(\omega) \cdot \mathbf{V}_{\omega}{ }^{T}(l)+\mathbf{n}_{l}, \\
& \mathbf{Y}=\mathbf{A}_{M}(\omega) \cdot \boldsymbol{\Theta}(\omega) \cdot\left(\mathbf{A}_{L}(\omega)\right)^{T}+\left[\mathbf{n}_{l}\right],
\end{aligned}
$$

where $\mathbf{n}_{l}=\left\{w_{\omega}(l), w_{\omega}(l+1), \ldots, w_{\omega}(l+M-1)\right\}^{T}$.

The problem of identifying the amplitude at a given frequency by sensing data $\mathbf{Y}$ can then be translated into finding the optimal filter problem. Define a regularized FIR filter $\mathbf{h}(\omega)$ through which only the component at a given frequency can pass, where $\mathbf{h}(\omega)=\left\{h_{i}(\omega)\right\}^{T}$ represents the filter parameter. $\mathbf{h}^{T}(\omega) \cdot \mathbf{Y}$ can be obtained using this filter to filter the observation matrix. The desired filtering effect is that the residual term $w_{\omega}(n)$ reaches its minimum value, that is, the noise reduction is zero, leaving only the contribution of signal components at other frequencies $\omega^{*}$ to it at frequency $\omega$. Meanwhile the filter does not distort the amplitude at the frequency $\omega$. That is to say,

$$
\min _{U(\omega) \mathbf{h}(\omega)}\left(\operatorname{mean}\left(\mathbf{h}^{T}(\omega) \cdot \mathbf{Y}-\mathbf{A}_{M}(\omega) \cdot \boldsymbol{\Theta}(\omega) \cdot\left(\mathbf{A}_{L}(\omega)\right)^{T}\right)\right)^{2} .
$$

The constraint is given as

$$
\mathbf{h}^{T}(\omega) \cdot \mathbf{A}_{M}(\omega)=\mathbf{c}^{T},
$$

where $\mathbf{c}=[1,0]^{T}$.

Equation (9a) is equivalent to maximizing the signal-tonoise ratio (SNR) of the filtered signal; that is,

$$
\max _{a(\omega), \mathbf{h}(\omega)} \frac{\left\|\mathbf{h}^{T}(\omega) \cdot \mathbf{A}_{M}(\omega)\right\|}{\mathbf{h}^{T}(\omega) \cdot \mathbf{Q}(\omega) \cdot \mathbf{h}(\omega)},
$$

where noise covariance is defined as $\mathbf{Q}(\omega)=E\left(\mathbf{n}_{l} \cdot \mathbf{n}_{l}\right)$, which can be replaced by its estimation $\widehat{\mathbf{Q}}(\omega)$ :

$$
\widehat{\mathbf{Q}}(\omega)=\frac{1}{L}\left[\mathbf{n}_{l}\right]\left[\mathbf{n}_{l}\right]^{T}=\widehat{\mathbf{R}}-\widehat{\mathbf{C}}(\omega) \cdot \mathbf{G}^{-1}(\omega) \cdot \widehat{\mathbf{C}}^{T}(\omega),
$$

where $\mathbf{G}(\omega)=(1 / L) \sum_{l=0}^{L-1} \mathbf{V}_{\omega}^{T}(l) \mathbf{V}_{\omega}(l) ; \widehat{\mathbf{C}}(\omega)=(1 / L) \sum_{l=0}^{L-1} \mathbf{y}_{l}$. $\mathbf{V}_{\omega}(l) ; \widehat{\mathbf{R}}$ is the estimation of the variance $\mathbf{R}$ :

$$
\widehat{\mathbf{R}}=E\left(\mathbf{y}_{l} \cdot \mathbf{y}_{l}^{T}\right)=\frac{1}{L} \mathbf{Y} \cdot \mathbf{Y}^{T} .
$$

The optimization problem can be further transformed into

$$
\begin{array}{ll}
\min _{U(\omega), \mathbf{h}(\omega)} & \mathbf{h}^{T}(\omega) \cdot \widehat{\mathbf{Q}}(\omega) \cdot \mathbf{h}(\omega), \\
& \mathbf{h}^{T}(\omega) \cdot \mathbf{A}_{M}(\omega)=[1,0]^{T} .
\end{array}
$$

Equation (9b) can be written as

$$
\mathbf{h}^{T}(\omega) \cdot \mathbf{A}_{M}(\omega) \cdot \widehat{\boldsymbol{\Theta}}(\omega)=\mathbf{c}^{T} \cdot \widehat{\boldsymbol{\Theta}}(\omega) ;
$$

that is,

$$
\mathbf{h}^{T}(\omega) \cdot \widehat{\mathbf{C}}(\omega) \cdot \mathbf{G}^{-1}=\widehat{\boldsymbol{\theta}}^{T}(\omega) .
$$

Taking the symmetry of $\mathbf{G}$ into consideration, we obtain

$$
\begin{aligned}
\widehat{\boldsymbol{\theta}}(\omega)= & \mathbf{G}^{-1}(\omega) \cdot \widehat{\mathbf{C}}^{T}(\omega) \cdot \mathbf{h}_{\mathrm{APES}}(\omega) \\
= & \mathbf{G}^{-1}(\omega) \cdot \widehat{\mathbf{C}}^{T}(\omega) \cdot \widehat{\mathbf{Q}}^{-1}(\omega) \cdot \mathbf{A}_{M}(\omega) \\
& \cdot\left(\mathbf{A}_{M}{ }^{T}(\omega) \widehat{\mathbf{Q}}^{-1}(\omega) \mathbf{A}_{M}(\omega)\right)^{-1} \cdot \mathbf{c},
\end{aligned}
$$

where $\widehat{\boldsymbol{\theta}}^{T}(\omega)$ is the estimation.

According to (3), the estimation of the FWAS and FWPhS can be given as follows:

$$
\begin{aligned}
& U(\omega)=\sqrt{U_{I}^{2}(\omega)+U_{\mathrm{Q}}^{2}(\omega),} \\
& \varphi(\omega)=\arctan \left(\frac{U_{I}(\omega)}{U_{\mathrm{Q}}(\omega)}\right) .
\end{aligned}
$$

2.3. Performance Comparison between APES and Fourier Transform in Signal Processing. Studies have shown that APES is a special form of the discrete Fourier transform (DFT), with a higher resolution in frequency and amplitude identification than the DFT. To compare the performance in spectrum identification of these two methods, a test signal is given, which consists of five harmonic waves at 5 different frequencies as shown in Table 1. The length of the signal is $600 \mathrm{~s}$, and the sampling frequency is $1 \mathrm{~Hz}$. A normal distribution noise with a mean of 0 and a standard deviation of 0.5 is added to the signal. The signal is processed using APES and the DFT, respectively, and the recognition results are shown in Table 1 and Figure 1.

As can be seen from Table 1 and Figure 1, the frequency components can be obtained at zero error using the APES method, while the DFT has a low resolution and a maximum error of up to $1.2 \%$. As for the amplitude recognition results, APES method also has obvious advantages of accuracy, and its maximum error is only $1.1 \%$. The DFT has a poor accuracy and its recognition result is generally less than the true value. The maximum error is $23.2 \%$ and the minimum error is $4.9 \%$. As shown in Figure 1, for the first two frequency components which are very close, the recognition results obtained from the DFT are almost coincident at the top, but the results obtained from APES have a large spread, indicating that APES has high frequency resolution.

It can be observed that not only an FWAS of high quality can be obtained using APES method, but also high frequency resolution. There are more frequency lines while 
TABLE 1: Amplitude spectral identified for test signal.

\begin{tabular}{|c|c|c|c|c|c|c|c|c|c|c|c|c|c|c|c|}
\hline \multirow{2}{*}{ Signals } & \multicolumn{3}{|c|}{1} & \multicolumn{3}{|c|}{2} & \multicolumn{3}{|c|}{3} & \multicolumn{3}{|c|}{4} & \multicolumn{3}{|c|}{5} \\
\hline & APES & Fourier & True & APES & Fourier & True & APES & Fourier & True & APES & Fourier & True & APES & Fourier & True \\
\hline \multirow{2}{*}{ Frequencies } & 0.053 & 0.05333 & \multirow{2}{*}{0.053} & 0.05599 & 0.05667 & \multirow{2}{*}{0.056} & 0.07706 & 0.07667 & \multirow{2}{*}{0.077} & 0.0805 & 0.08 & \multirow{2}{*}{0.0805} & 0.09306 & 0.09333 & \multirow{2}{*}{0.093} \\
\hline & $\begin{array}{ll}S & 0\end{array}$ & $0.62 \%$ & & 0 & $1.2 \%$ & & 0 & $0.4 \%$ & & 0 & $0.6 \%$ & & 0 & $0.4 \%$ & \\
\hline \multirow{2}{*}{ Amplitude } & 12.34 & 9.985 & \multirow{2}{*}{12.3} & 10.06 & 8.313 & \multirow{2}{*}{10} & 5.353 & 5.813 & \multirow{2}{*}{5.3} & 10 & 7.777 & \multirow{2}{*}{10} & 10.11 & 9.512 & \multirow{2}{*}{10} \\
\hline & $0.3 \%$ & $23.2 \%$ & & $0.6 \%$ & $16.9 \%$ & & $1 \%$ & $9.68 \%$ & & 0 & $22.2 \%$ & & $1.1 \%$ & $4.9 \%$ & \\
\hline $\mathrm{NP}^{*}$ & & 31.8 & & & 33.6 & & & 46.2 & & & 48.3 & & & 55.8 & \\
\hline
\end{tabular}

Note. NP is number of periods.

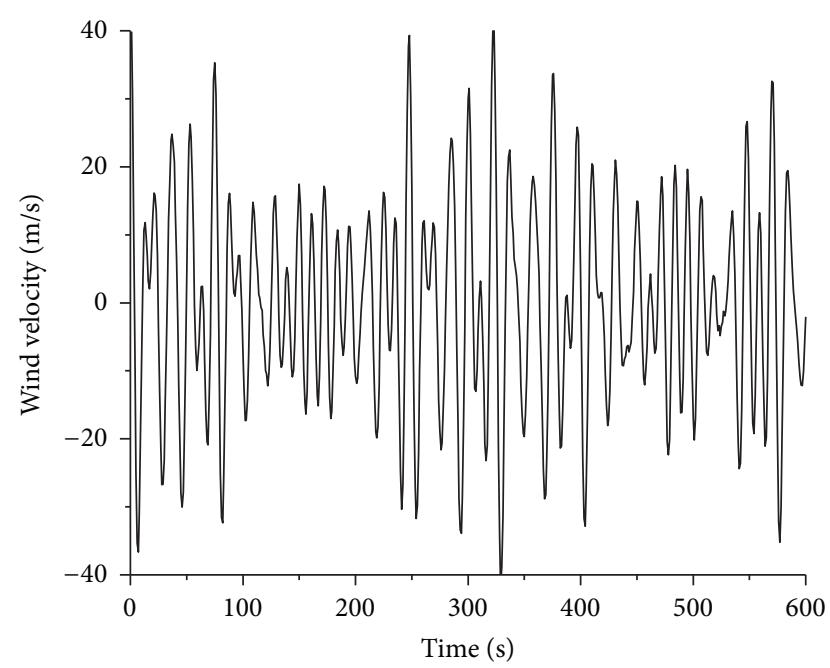

(a) Time history of the test signal

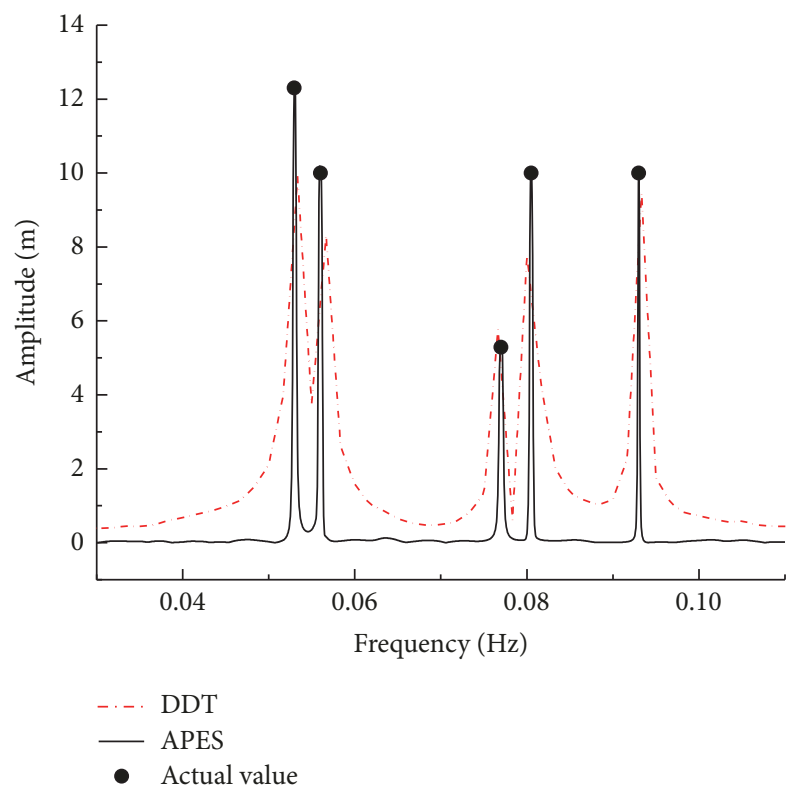

(b) Amplitude spectra identified by DFT and APES

FIGURE 1: Test signal and the identified result.

using APES, which makes the spectra distribution more realistic. In addition, since the usual time for wind spectrum estimation is $600 \mathrm{~s}$, there are only 31.8 periods for the first recognized frequency, and, in such a short period, APES method is more reliable than the DFT.

\section{FWAS Obtained Based on Measured Wind Velocity Data Using APES Method}

Shanghai Minpu Second Bridge is a double-deck large bridge for both highway and railway (as shown in Figure 2) located in the upper reaches of Shanghai Huangpu River, MinhangFengxian section. The main bridge is a single tower doubledecked cable-stayed bridge with double cable planes, with a main span of $251.4 \mathrm{~m}$, and anchor span of $147 \mathrm{~m}+$ $38.25 \mathrm{~m}$. The upper deck is a secondary highway with twoway four lanes, at a width of $18 \mathrm{~m}$, while the lower deck is a two-track light railway (Fengxian section of Line 5 in Shanghai), with a minimum functional width of $10 \mathrm{~m}$. The monitoring contents of Minpu Second Bridge Structural Health Monitoring System were selected considering the bridge structural characters. Furthermore, many types of monitoring objectives are adopted. There are 172 measuring points; two of these are wind velocity and wind direction measuring points (as shown in Figure 2) located at the top of the tower and in the middle of the main span, respectively. The top of the tower is $150 \mathrm{~m}$ and the middle of the main span is $29 \mathrm{~m}$ (Wusong zero elevation), which are less than the local gradient wind height. The sampling frequency of the anemometer is $4 \mathrm{~Hz}$ and uninterrupted sampling was used to monitor the wind speed acting on the structure.

The monitoring of wind velocity data from January 2016 to March 2016 is divided into 13,104 samples, which has a time interval of 10 minutes and consists of two groups of data at the top of the tower and in the middle of the span, according to the Wind-Resistant Design Specification for Highway Bridges (JTG/T D60-01-2004) [18] published by the Ministry of Communications in China.

The main wind velocity, $\bar{u}$, the horizontal wind velocity, $\bar{u}_{p}$, and the horizontal mean wind direction, $\varphi$, are calculated by the vector decomposition method [19]:

$$
\begin{aligned}
\bar{u} & =\sqrt{\bar{u}_{x}{ }^{2}+\bar{u}_{y}{ }^{2}+\bar{u}_{z}{ }^{2}}, \\
\bar{u}_{p} & =\sqrt{\bar{u}_{x}{ }^{2}+\bar{u}_{y}{ }^{2}}
\end{aligned}
$$



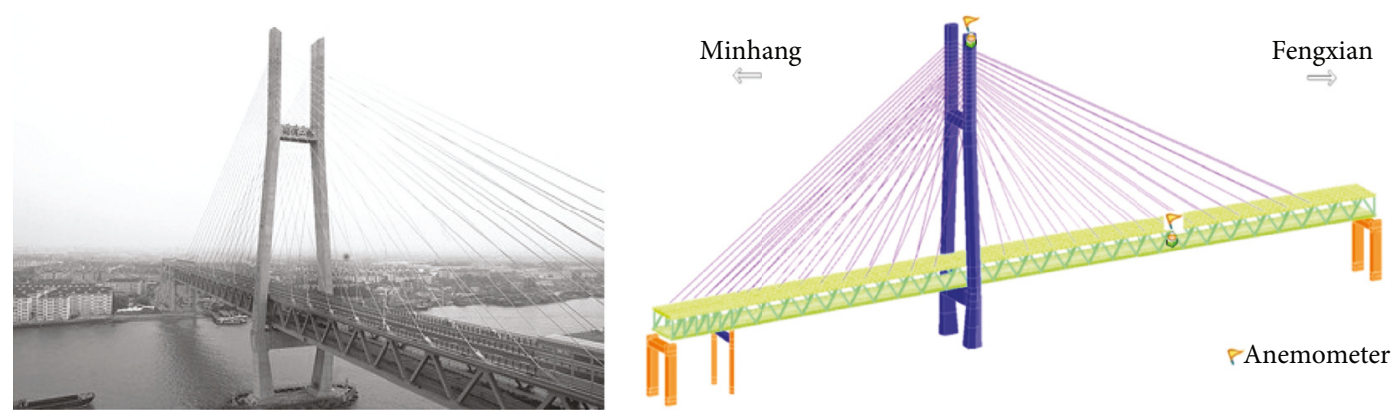

Figure 2: Aerial view of Minpu Second Bridge and the layout of anemorumbometers.

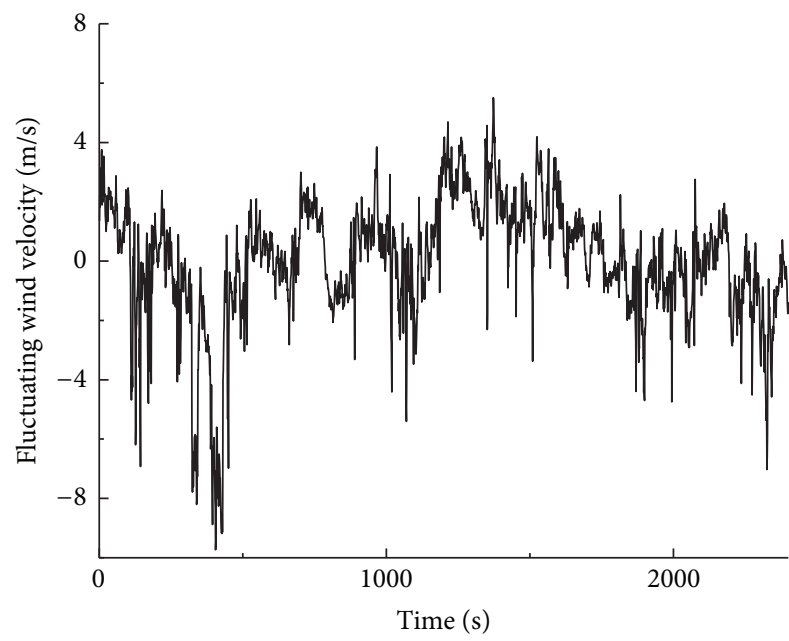

(a)

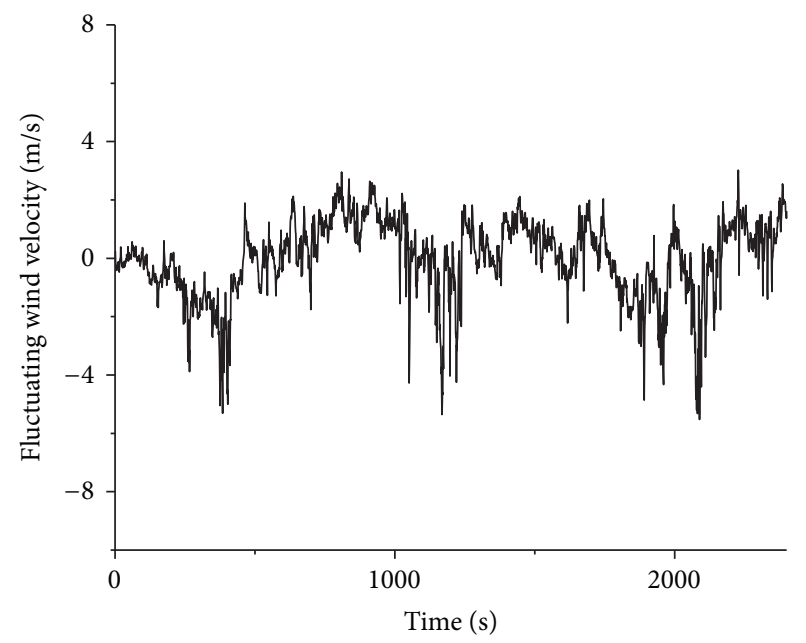

(b)

FIGURE 3: Typical fluctuating wind velocity time history.

$$
\begin{aligned}
& \cos (\varphi)=\frac{\bar{u}_{x}}{\bar{u}_{p}} \\
& \sin (\varphi)=\frac{\bar{u}_{y}}{\bar{u}_{p}},
\end{aligned}
$$

where $\bar{u}_{x}, \bar{u}_{y}$, and $\bar{u}_{z}$ represent the mean wind velocity in the lengthways and transverse and vertical direction, respectively. As mentioned above, the sampling frequency is $4 \mathrm{~Hz}$ and the time interval is $600 \mathrm{~s}$; therefore these parameters can be written as

$$
\begin{aligned}
& \bar{u}_{x}=\frac{1}{2400} \sum_{i=1}^{2400} u_{x}\left(t_{i}\right), \\
& \bar{u}_{y}=\frac{1}{2400} \sum_{i=1}^{2400} u_{y}\left(t_{i}\right), \\
& \bar{u}_{z}=\frac{1}{2400} \sum_{i=1}^{2400} u_{z}\left(t_{i}\right) .
\end{aligned}
$$

Before estimating the wind spectra, the data samples should be filtered in accordance with the following principles [20]:

(1) The number of the data points of the raw data obtained from the two monitoring sites, which are over $64 \mathrm{~m} / \mathrm{s}$, should be less than 50 .

(2) The main wind velocity $\bar{u}$ in both sites is over $6 \mathrm{~m} / \mathrm{s}$.

(3) The main wind velocity $\bar{u}$ in the middle of the span is less than that at the top of the tower.

(4) The horizontal wind velocity $\bar{u}_{p}$ in the middle of the span is less than that at the top of the tower.

After filtering, 1,014 data samples that meet the requirements of stationary wind were obtained. The time history of each fluctuating wind sample is derived as follows:

$$
u(t)=u_{x}(t) \cos (\varphi)+u_{y}(t) \sin (\varphi)-\bar{u}_{p}
$$

Two instances of fluctuating wind time history are shown in Figure 3. 

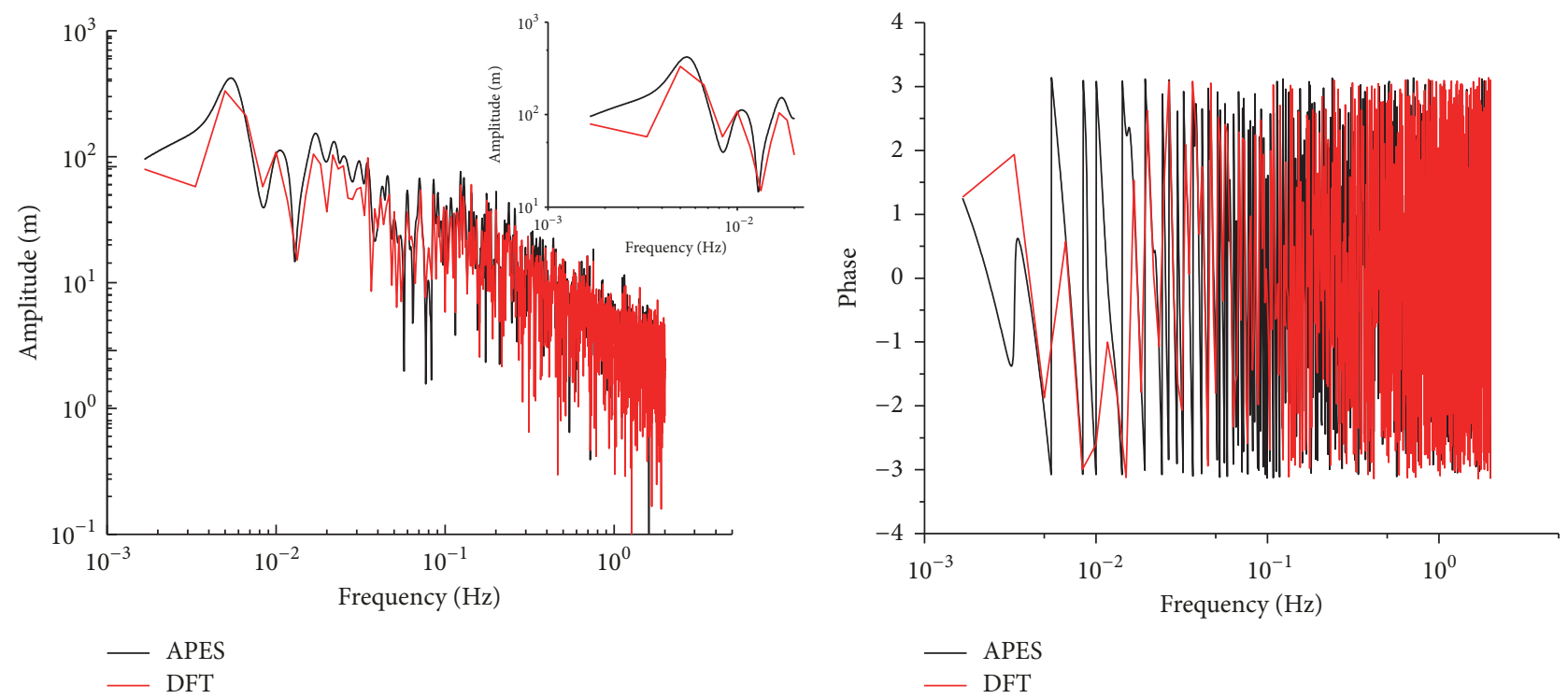

FIgURE 4: FWAS and FWPhS estimation of a certain sample.

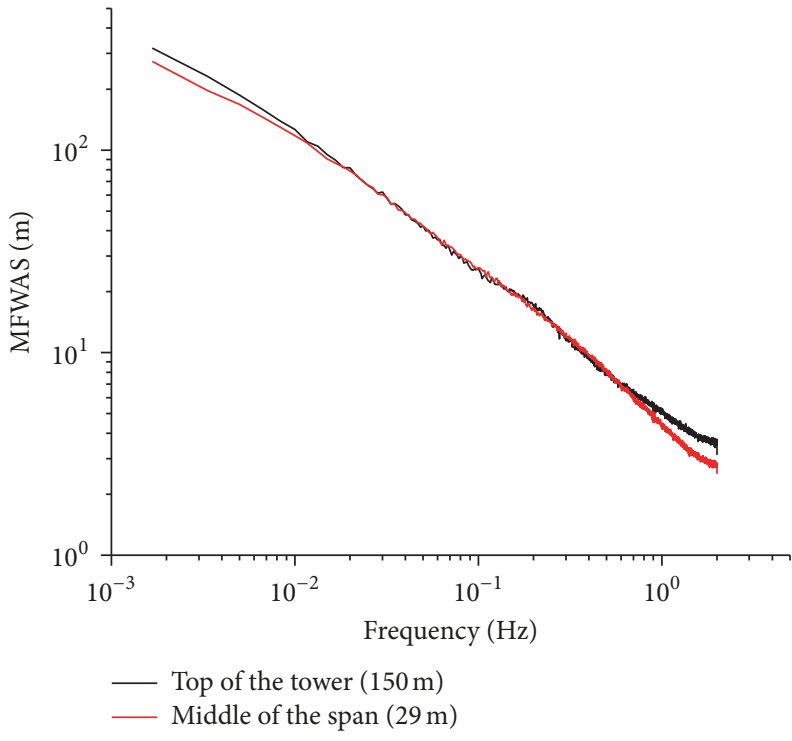

(a) DFT

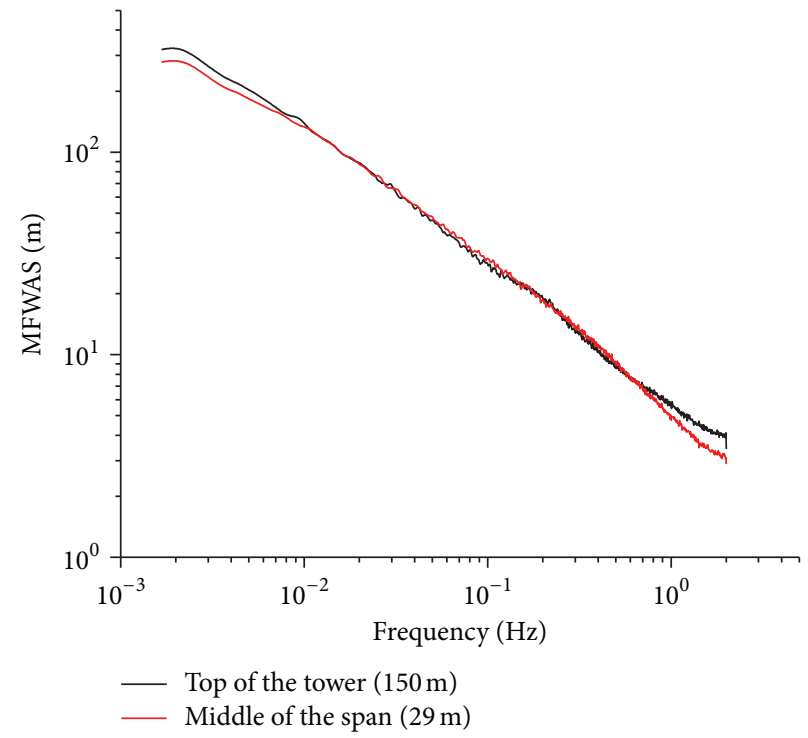

(b) APES

FIGURE 5: Mean of measured FWASs obtained using the two methods.

3.1. Estimation of FWAS and FWPhS of Stationary Fluctuating Wind. The FWAS and FWPhS of 1014 samples are estimated using the APES method and DFT, respectively. The FWAS and FWPhS of one sample are shown in Figure 4. As shown in Figure 4, in the logarithmic axis of frequency, the FWAS curve calculated by APES is smoother than that calculated by the DFT, and there are more frequency points calculated by APES in low-frequency region. The frequency points in high frequency region are more intensively calculated by the DFT, which covers the overall trend of the spectral line. In conclusion, the spectral line resolution and accuracy calculated by APES is higher than that calculated by the DFT because the spectrum at any given frequency can be estimated by APES method; therefore, the frequency points can be distributed evenly in logarithmic axis. However, while using the DFT, the time history is projected onto the frequency points, which are set in advance. Furthermore, the frequency point calculated by the DFT is an arithmetic sequence, which results in the lack of frequency points in low-frequency region and intensive frequency points in high frequency region.

Statistical analysis for the 1014 data samples was performed using APES and the DFT. The mean and standard deviation of the measured FWASs of the middle span and the tower top obtained using the two methods are given in Figures 5 and 6, respectively, and the difference in the 


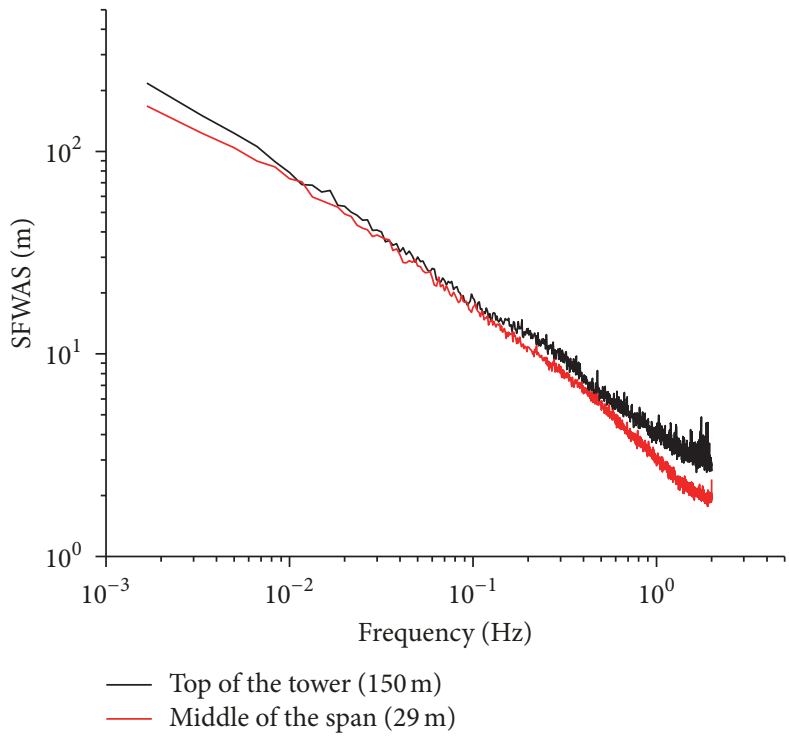

(a) DFT

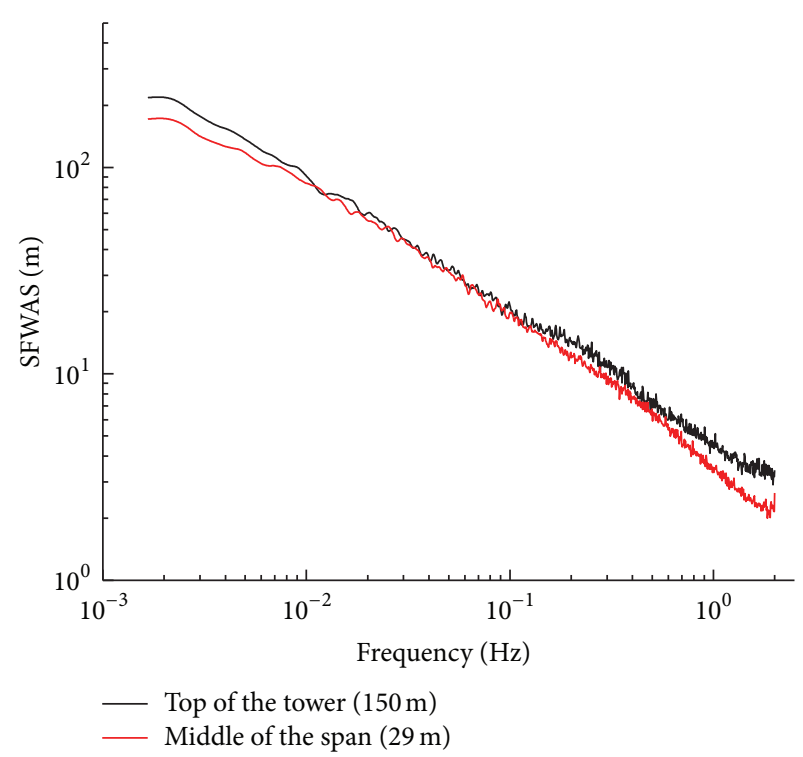

(b) APES

FIGURE 6: Standard deviation of measured FWASs obtained using the two methods.

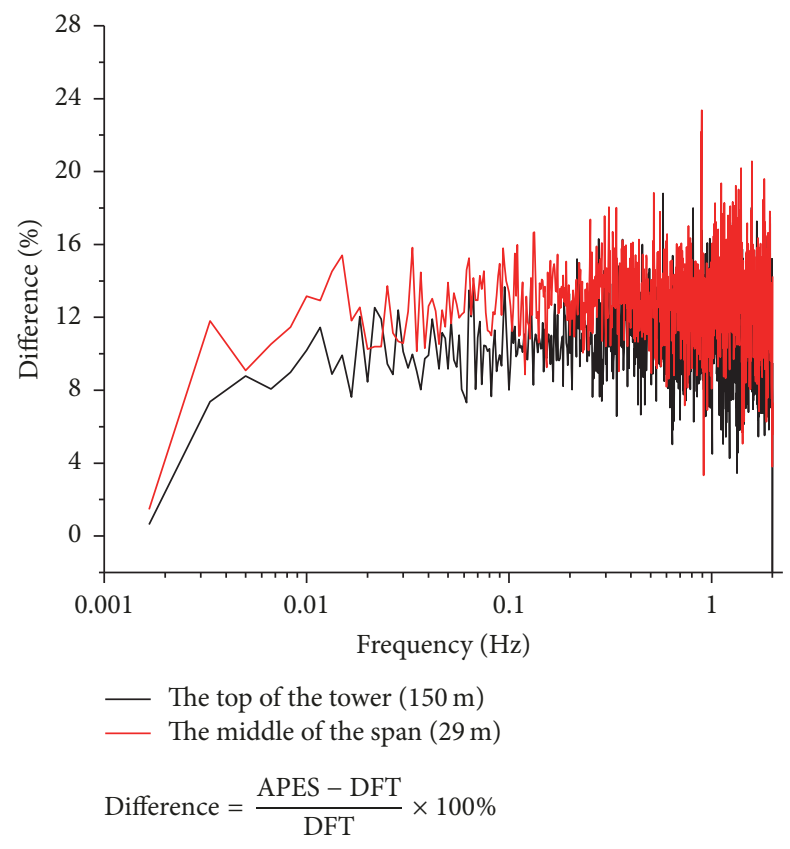

Figure 7: Difference between the two means of measured FWASs.

mean FWAS using the two methods is given in Figure 7. The mean of FWPhSs is given in Figure 8, and the corresponding histogram is given in Figure 9.

As shown in Figures 5-9

(1) The results obtained by the two methods are consistent: the amplitude in low-frequency region is high and low in high frequency region. In the double logarithmic coordinate system, the spectral trend decreases linearly, which is similar to results found in literature, that is, the energy of the fluctuating wind is concentrated in the low-frequency region. The mean of the FWAS in the middle of the span is smaller than that at the top of the tower in the low-frequency and high frequency regions but nearly the same in intermediate frequency region.

(2) The standard deviation of the measured FWASs obtained by the two methods is high in low-frequency region and small in high frequency region, which indicates that turbulence energy is concentrated in low-frequency region from the side.

(3) The mean of the FWAS obtained using the DFT is smaller than that obtained using APES in the full frequency section. The maximum difference was up to $23.37 \%$, and the minimum difference was $0.63 \%$, which is consistent with the results presented in Section 2.3. Therefore, we can conclude that the results obtained using APES are more accurate.

(4) The distribution of the FWPhSs obtained using the two methods is consistent, with a mean value of 0 , and the frequency of every phase interval is roughly equal, which indicates that the FWPhS is almost consistent and evenly distributed with a mean value of 0 in $[-\pi, \pi]$. 


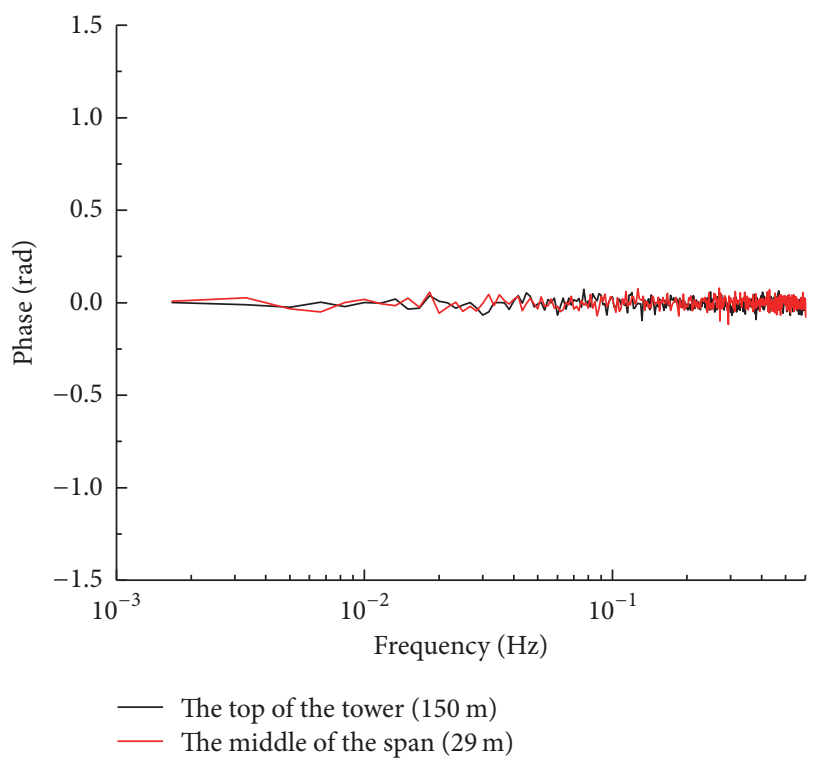

(a) DFT

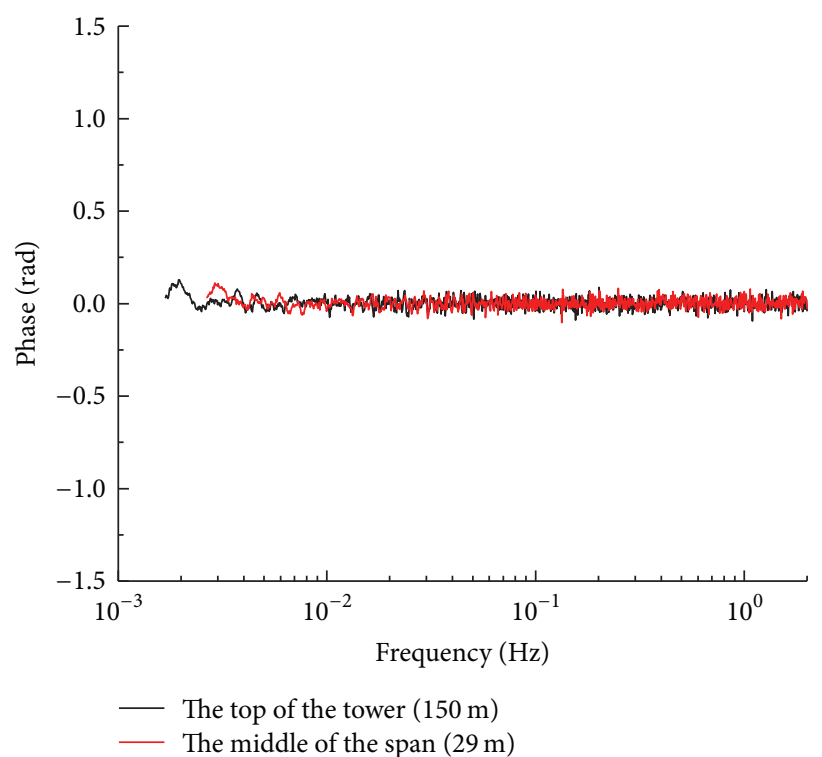

(b) APES

FIGURE 8: Mean of FWPhS obtained using the two methods.

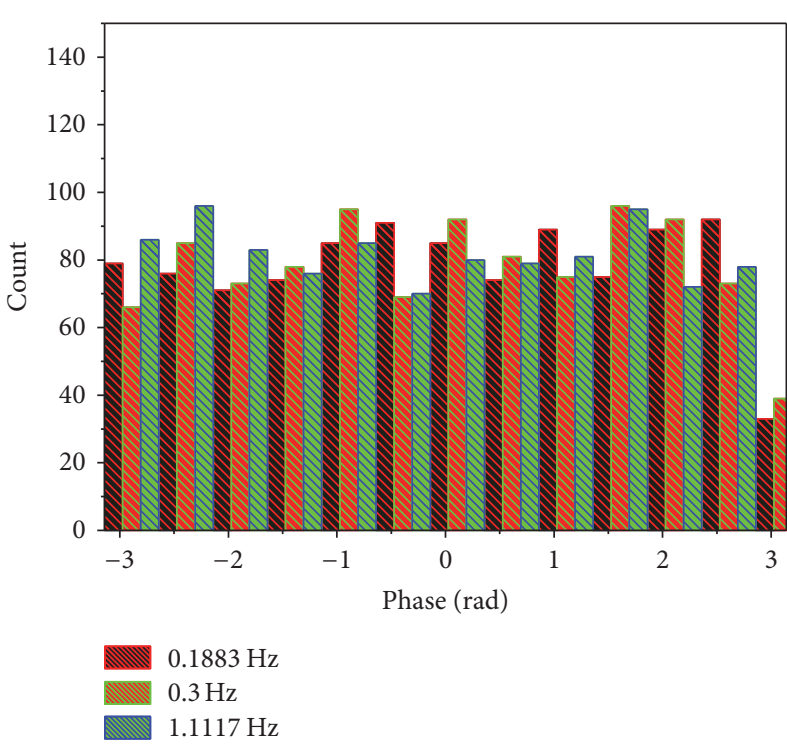

(a) DFT

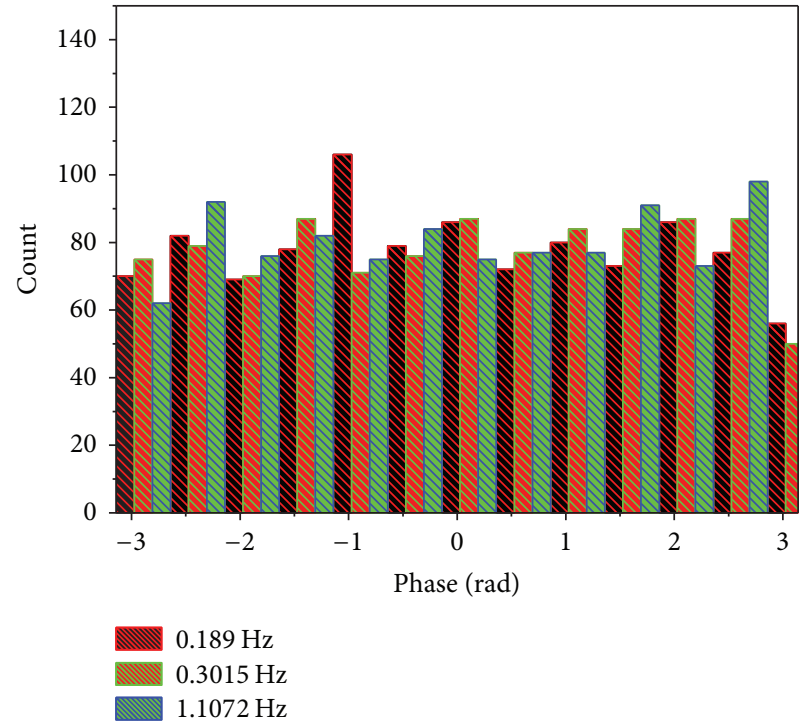

(b) APES

FIgURE 9: Phase histogram obtained using the two methods.

\section{FWAS Model Based on Measured Data}

4.1. Empirical Expression of FWAS. According to the Kolmogorov theory, the fluctuating wind power spectrum (FWPS) can be written as the following empirical expression:

$$
S(z, \omega)=\frac{A \cdot 2 \pi u_{*}^{2} f^{\gamma}}{\omega\left(1+B f^{\alpha}\right)^{\beta}},
$$

where $S(z, \omega)$ represents the FWPS at the height $z, A$ and $B$ are constants, and $\alpha, \beta$, and $\gamma$ are power exponents satisfying $\alpha \beta-\gamma=2 / 3$. The friction rate $u_{*}$ can be obtained as follows:

$$
u_{*}=\frac{u_{z} k}{\ln \left(z / z_{0}\right)},
$$

where $u_{z}$ is the mean wind velocity at the height $z$ and $z_{0}$ is the ground roughness. $k$ is Von Karman constant and is 


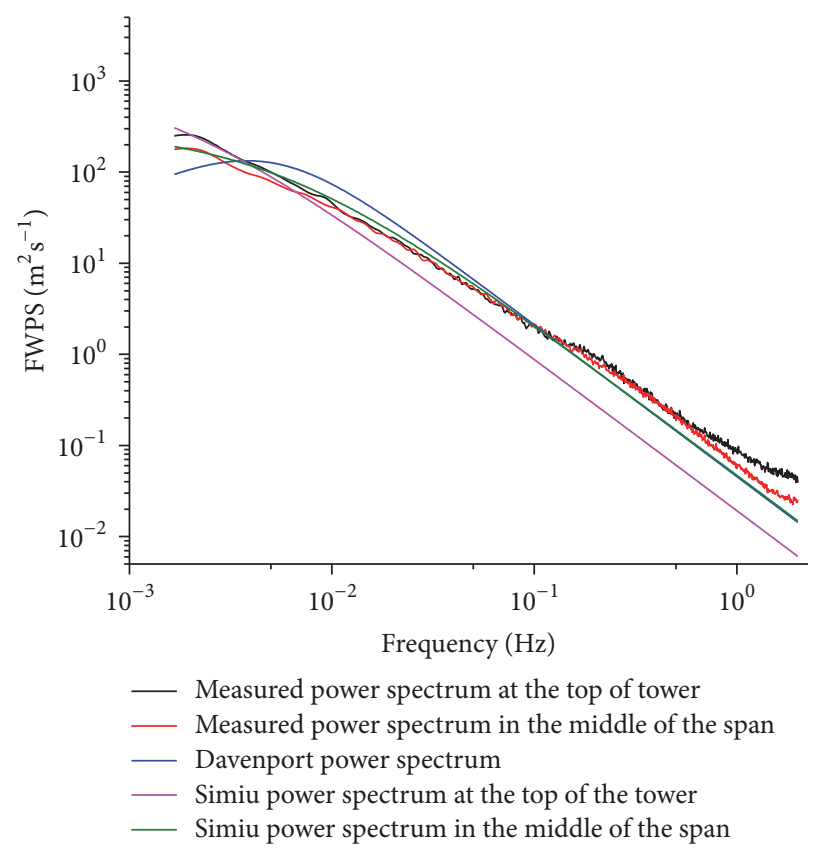

Figure 10: Measured FWPS and eFWPSs given in specifications.

usually set to $0.4 ; f$ is Molin coordinate often expressed in the following form:

If $f=L^{2} \omega /\left(2 \pi u_{10}\right)$, the FWPS obtained corresponds to Von Karman power spectrum and $L$ is turbulence integral scale, where $u_{10}$ is the mean wind velocity at the height of $10 \mathrm{~m}$.

If $f=1200 \omega / 2 \pi u_{10}$, the FWPS obtained corresponds to Davenport power spectrum.

If $f=\omega z /\left(2 \pi u_{z}\right)$, the FWPS obtained corresponds to Simiu power spectrum.

According to the definition of the power spectrum and amplitude spectrum of stationary stochastic signal, the relationship between the unilateral power spectrum $S(z, \omega)$ and unilateral amplitude spectrum $U(z, \omega)$ is given as

$$
S(z, \omega)=\frac{1}{T} E\left[U^{2}(z, \omega)\right],
$$

where $T$ is $600 \mathrm{~s}$ according to the specifications of China.

Define the mean of the FWAS as $E(U(z, \omega))$, and the standard deviation of the FWAS as $\operatorname{Std}(U(z, \omega))$. Apparently, $U^{2}(z, \omega), E(U(z, \omega))^{2}, \operatorname{Std}(U(z, \omega))^{2}$, and the mean square expectation of FWAS $E\left[U^{2}(z, \omega)\right]$ have the same dimension; that is, $U(z, \omega), E(U(z, \omega)), \operatorname{Std}(U(z, \omega))$, and $\sqrt{T S(z, \omega)}$ have the same dimension. Thus, according to (21), the unified expression of the empirical FWAS (for convenience, the eFWAS, also expressed as $U(z, \omega))$ can be expressed as follows:

$$
U(z, \omega)=\frac{C u_{*} f^{0.5 \gamma}}{\sqrt{\omega}\left(1+B f^{\alpha}\right)^{0.5 \beta}},
$$

where $f$ is consistent with (21); when $f$ takes different forms, (24) corresponds to different types of FWASs. Friction rate $u_{*}$ and Molin coordinate $f$ are random variables. Therefore, the FWAS is a random variable. The empirical forms of $U(z, \omega)$ can be fitted using measured data.

4.2. The Specific Expression of Empirical Fluctuating Wind Model. The specific expression of the eFWAS depends on the selection of Molin coordinate, and it can be selected by comparing it with the mean value and deviation of measured FWAS. However, the coefficients in (24) are unknown, and we cannot compare the selected expression with the measured values. On the other hand, the existing fluctuating wind models are in the form of power spectrum. The parameters of most fluctuating wind models have been determined by many researchers, and some have been adopted as the standard models in the specifications. Thus, the proper expression of the eFWAS can be selected by comparing the measured FWPS with the empirical FWPSs (eFWPSs) given in the specifications.

The Davenport eFWPS, adopted in Canadian specification [21], and Simiu eFWPS, adopted in Chinese specification [18], are compared with the measured FWPS as shown in Figure 10.

As shown in Figure 10, there is a slight difference in the measured FWPSs in both sites in low-frequency region and high frequency region, but no difference in other frequency region, which indicates that the fluctuating wind field is independent of height in this area. This is the same as the regularity of Davenport power spectrum, which is independent of height, and, except for the large error in low-frequency, the measured FWPS coincides with the Davenport eFWPS 
in the other frequency region. Figure 10 also shows that Simiu eFWPS is related to height and varies widely from the measured FWPS at the top of the tower, although it is close to the measured FWPS in the middle of the span.

It is foreseeable that anastomosis degree between Davenport eFWPS and measured FWPS can be improved by adjusting the parameters of Davenport eFWPS expression, such that the Davenport eFWPS can describe the FWPS in this area. However, no matter how the parameters of Simiu eFWPS were adjusted, the Simiu eFWPS cannot express the regularity that the measured FWPS is independent of height. The above shows that Davenport eFWPS is more suitable to describe the FWPS character in this area; this is also true for the Davenport eFWAS. In this paper, considering the Davenport eFWAS, the parameters are estimated using the measured FWAS to establish an empirical fluctuating wind model, which is suitable for the wind field in the bridge site.

4.3. Determination of Parameters of Davenport eFWAS. The Molin coordinate is defined as $f=1200 n / u_{10}$, where $n=$ $\omega / 2 \pi$. Substituting (22) into (24), the expression for the Davenport eFWAS is as follows:

$$
U(n)=\frac{C_{1} k u_{10}\left(1200 n / u_{10}\right)^{C_{3} C_{4}-1 / 3}}{\ln \left(10 / z_{0}\right)\left[1+C_{2}\left(1200 n / u_{10}\right)^{C_{3}}\right]^{C_{4}} \sqrt{n}} .
$$

$U(z, \omega)$ is abbreviated as $U(n)$ because it is independent of height. For convenience, the parameters $C, B, \gamma, \alpha$, and $\beta$ in (23) are rewritten as $C_{1}, C_{2}, C_{3}$, and $C_{4}$.

The parameter estimation of the eFWAS is essentially a parameter optimization problem, that is, to find a set of parameters to make the error between the mean of the eFWAS and mean of the measured FWAS and between the standard deviation of the eFWAS and standard deviation of measures FWAS as small as possible. Thus, define the spectral error as

$$
\Delta=\left|\widehat{U}^{m}(n)-E(U(n))\right|+\left|\widetilde{U}^{m}(n)-\operatorname{Std}(U(n))\right|,
$$

where $\widehat{U}^{m}(n)$ and $\widetilde{U}^{m}(n)$ represent the mean value and standard deviation of the measured FWAS, respectively, and $E(U(n))$ and $\operatorname{Std}(U(n))$ represent the mean value and standard deviation of the eFWAS obtained when $u_{10}$ and $z_{0}$ randomly changed.

Particle swarm optimization (PSO) is used to optimize the multiparameter problem, which is a simple but efficient method. The parameter sets $C_{1}, C_{2}, C_{3}$, and $C_{4}$ are obtained after several optimization iterations. If we substitute the parameter set into (25), the final Davenport eFWAS model is obtained as follows:

$$
U(n)=\frac{601.4 u_{10}^{0.6138} n^{-0.1138}}{\ln \left(10 / z_{0}\right)\left[1+19.28\left(n / u_{10}\right)^{0.3846}\right]^{1.8709}}
$$

The eFWAS model given in (27) can express at least the firstand second-order probability properties of the real FWAS because (27) is in line with the mean value and standard deviation of the measured FWAS. The stochastic wind information, including mean value and standard deviation, can be obtained using (27), which is the first application of the empirical fluctuating wind model.

It is difficult to obtain the statistical information of $u_{10}$ and $z_{0}$ in engineering practice but relatively easy to obtain the mean value of $u_{10}$ and $z_{0}$, denoted as $\widehat{u}_{10}$ and $\widehat{z}_{0}$.

Define eFWAMS and eFWASS as the fluctuating wind mean amplitude spectrum and amplitude standard deviation spectrum, and, in (25), $u_{10}$ and $z_{0}$ are replaced with $\widehat{u}_{10}$ and $\widehat{z}_{0}$. PSO is also used to optimize the parameter set with the goal of making the error between mean of the measured FWAS and eFWAMS (see (28)) or standard deviation of the measured FWAS and eFWASS (see (29)) as small as possible.

$$
\begin{aligned}
& \widehat{U}(n)=\frac{1300 \cdot \widehat{u}_{10}^{0.4876} \cdot n^{0.5124}}{\ln \left(10 / \widehat{z}_{0}\right)\left[1+47.04\left(n / \widehat{u}_{10}\right)^{0.4750}\right]^{1.7805}}, \\
& \widetilde{U}(n)=\frac{2704 \cdot \widehat{u}_{10}^{0.4029} \cdot n^{0.5971}}{\ln \left(10 / \widehat{z}_{0}\right)\left[1+17.82\left(n / \widehat{u}_{10}\right)^{0.4006}\right]^{2.3225}} .
\end{aligned}
$$

The main probability properties of stochastic wind field can be obtained through (28) and (29), which is the second application of the empirical fluctuating wind model, that is, the combination of eFWAMS and eFWASS.

4.4. Probability Properties of Davenport eFWAS. Equation (27) is a stochastic spectrum model obtained by fitting its mean value and standard deviation with the mean value and standard deviation of the measured FWAS, and its randomness comes from the random variables $u_{10}$ and $z_{0}$. The fit degree can be investigated by comparing its mean value, standard deviation, and data distribution at certain frequencies. Substituting the 1014 set of $u_{10}$ and $z_{0}$ into (27), 1014 amplitude spectra are obtained, while the mean value and standard deviation of the 1014 spectra are also obtained. Furthermore, the eFWAMS and eFWASS can be obtained according to (27) and (29).

The mean value of the eFWAS, the eFWAMS, and the mean value of the measured FWAS are plotted in Figure 11(a), while the standard deviation of the eFWAS, the eFWASS, and the standard deviation of the measured FWAS are plotted in Figure 11(b). The plots show good agreement, which indicates that a high-quality mean of the FWAS can be obtained using (27) or (28) and the standard deviation of the FWAS can be obtained using (27) or (28). The comparison of results shows that the eFWAS presented in this paper is consistent with measured values with respect to the first- and second-order moment.

A plot of $\delta$, defined as the ratio of the eFWAMS to eFWASS, is shown in Figure 11(c). The ratio is virtually unchanged in the frequency domain with a mean value of 1.8847. This indicates that the mean and the approximate standard deviation (dividing the mean value by 1.8847 ) of 


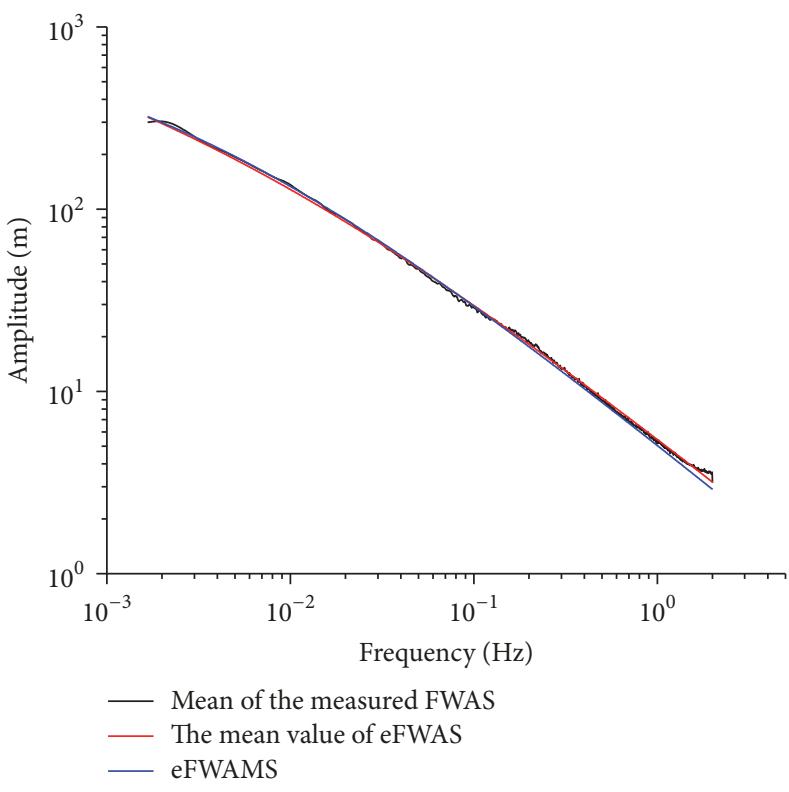

(a)

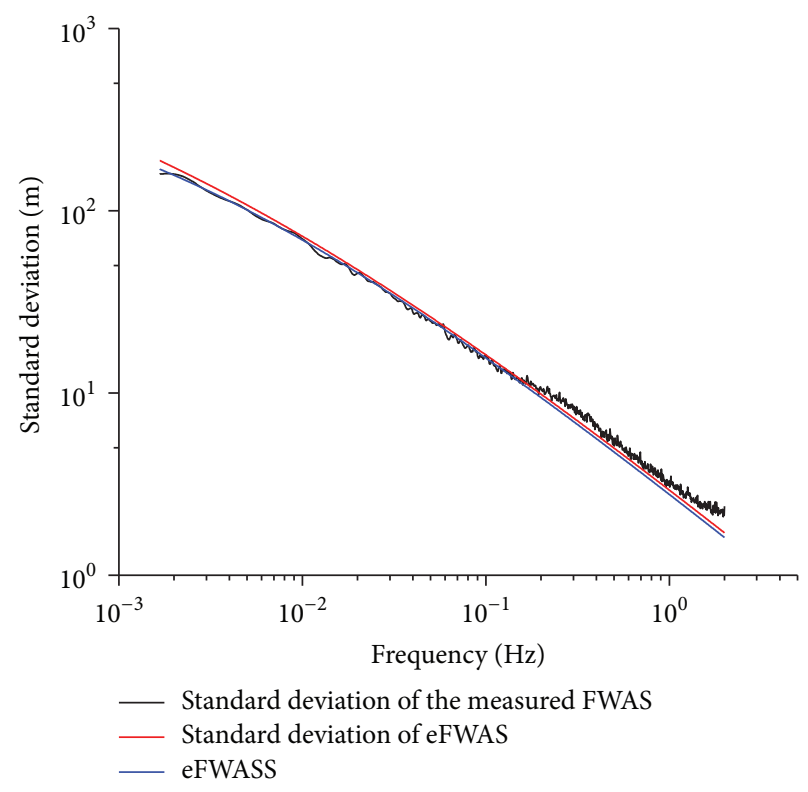

(b)

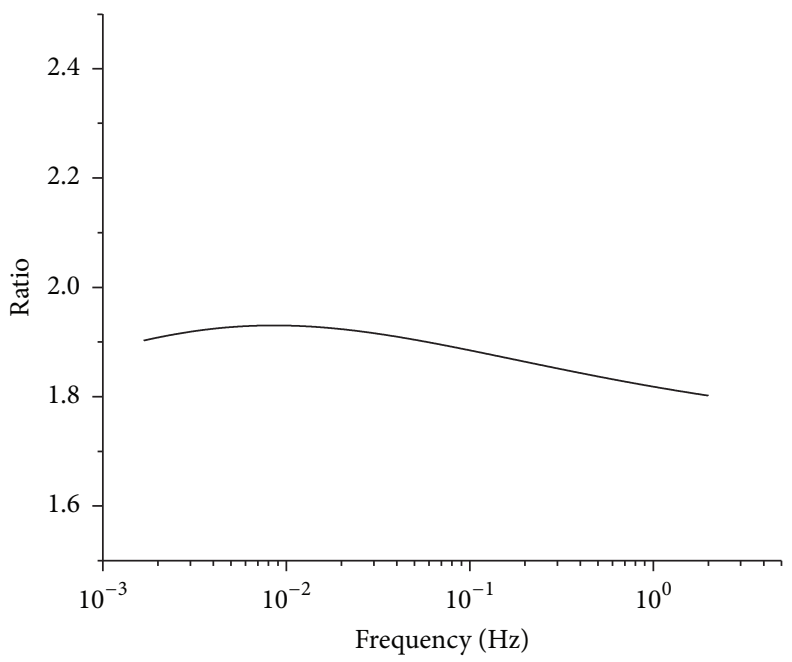

(c)

FIGURE 11: Comparison of results for eFWAMS and eFWASS.

the FWAS can be obtained only by (28). This is the third application of the empirical fluctuating wind model.

Further study of the distribution of the eFWAS is investigated. The histograms of amplitude distribution at frequency values of $0.005,0.1$, and $1 \mathrm{~Hz}$ are plotted in Figure 12, representing the amplitude distribution at low, medium, and high frequency regions. In Figure 12, the horizontal axis is dimensionless and defined as the ratio of amplitude to its mean value. As shown in these three figures, the amplitude distribution of both the eFWAS and measured FWAS is consistent with the lognormal distribution.

To further investigate the amplitude distribution at the entire frequency region, the mean value and mean value plus and minus one time deviation of both the eFWAS and measured FWAS are plotted in Figure 13. As shown in the figure, the three spectral lines of the eFWAS are consistent with the measured FWAS, which is an indication that the eFWAS model proposed in this paper can accurately express the FWAS probability distribution law in the entire frequency region.

4.5. Summary of Empirical Fluctuating Wind Model Concept System. So far, we have developed a series of empirical expressions to describe the amplitude of random fluctuating wind speed. In order to give a full view for these models, a framework block diagram for all the concepts employed in this paper is illustrated in Figure 14. The three applications methods proposed in this paper are also shown in this figure. 


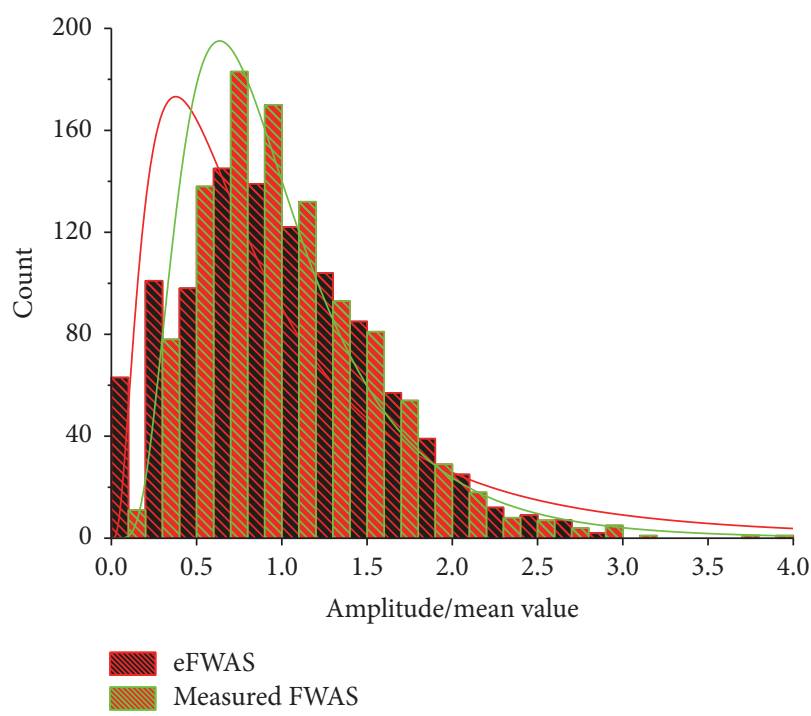

(a) $0.005 \mathrm{~Hz}$

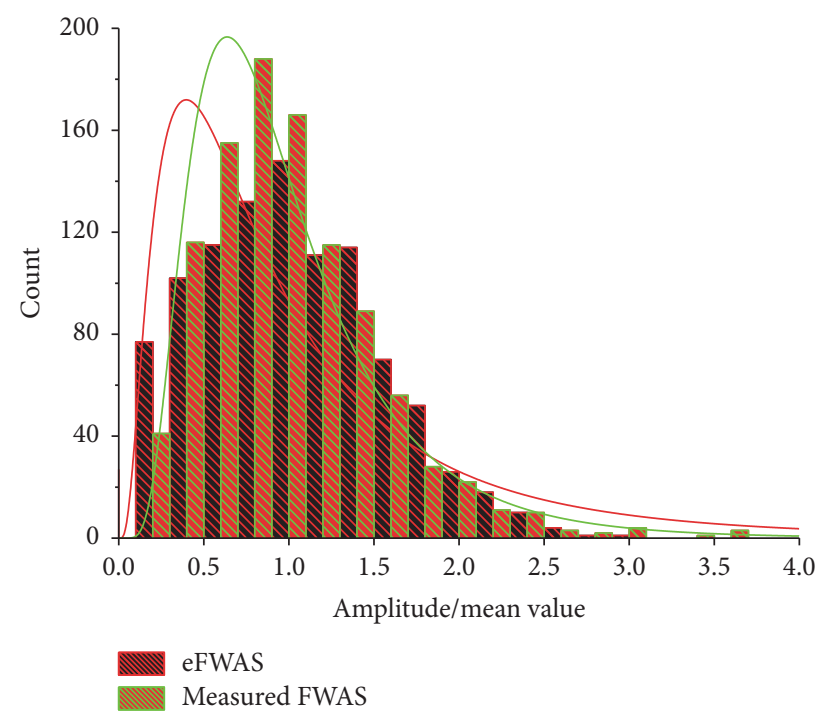

(b) $0.1 \mathrm{~Hz}$

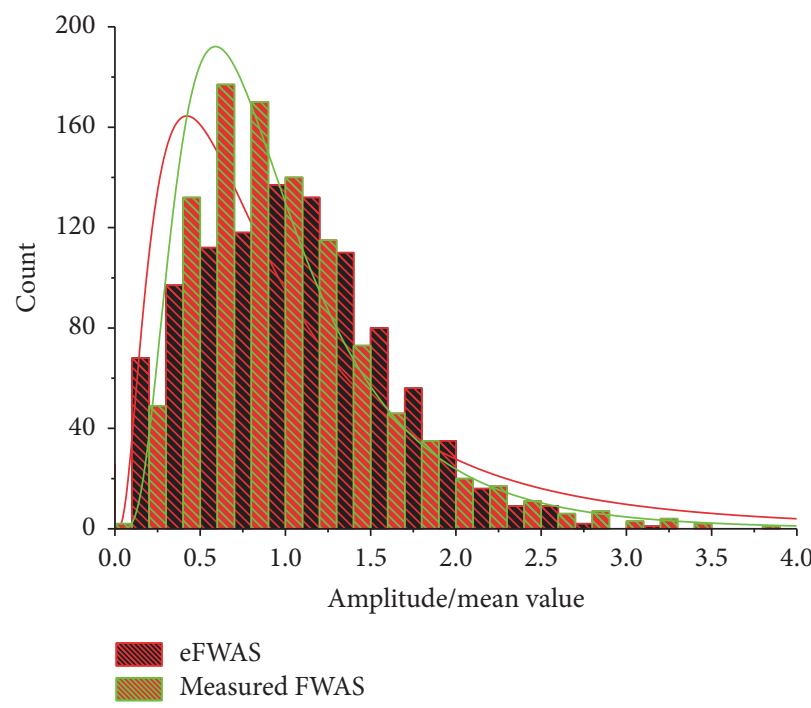

(c) $1 \mathrm{~Hz}$

Figure 12: Amplitude distribution at certain frequencies.

\section{Conclusion}

In the analysis of effects induced by wind load and antiwind structural design, inaccurate analysis results and inappropriate design are obtained because of inappropriate wind spectrum. With respect to the wind field in a specific area, the FWAS obtained using measured wind velocity data is more accurate and more specific. In this paper, a highresolution nonparametric spectral analysis method (APES) is adopted, and the measured FWAS, mean of the measured FWAS, standard deviation of the measured FWAS, and FWPhS were estimated, which were of higher quality and resolution compared with the spectra obtained using the DFT. Through dimensional analysis, the eFWAS model is proposed and the parameters of the Davenport eFWAS were obtained using the measured FWAS. Three specific applications of the eFWAS model are proposed, which can be used under different conditions. Probability properties studies proved that the eFWAS model proposed in this paper can describe the complete statistical information of the wind field from the first-order moment, second-order moment, and the amplitude distribution, which is beyond the capability of FWPS. This is important in terms of improving the wind load modeling resolution, antiwind performance of new structures, and accurate assessment of safety and wind resistance of structures.

\section{Conflicts of Interest}

The authors declare that there are no conflicts of interest regarding the publication of this paper. 


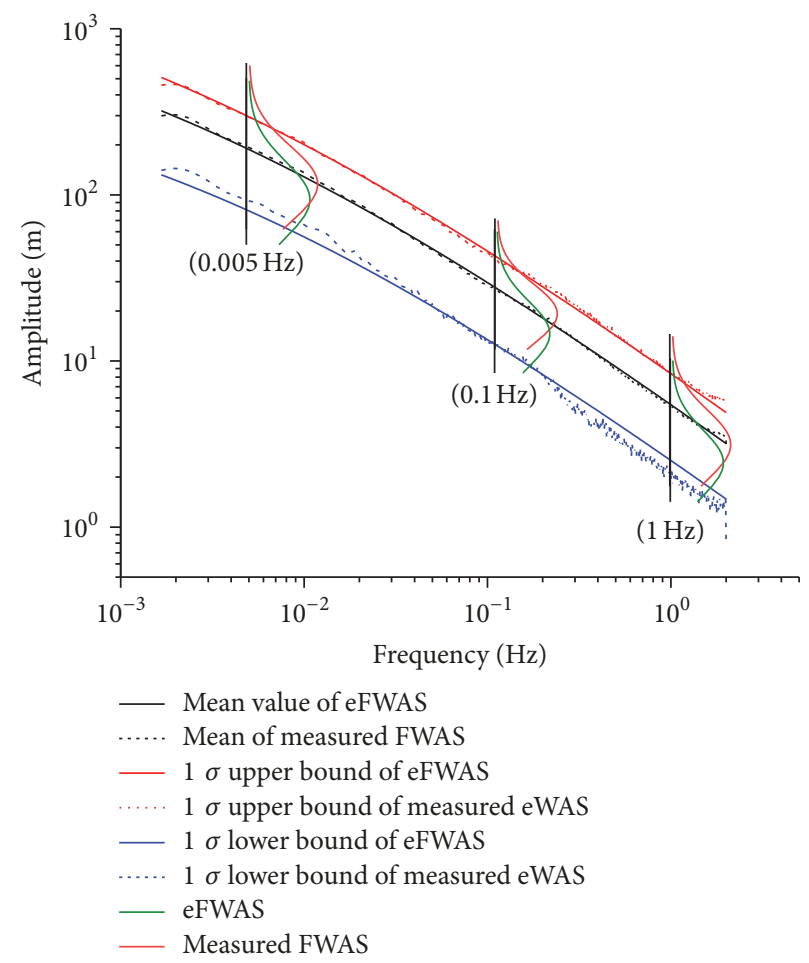

FIGURE 13: Statistical characteristic comparison of eFWAS and measured FWAS.

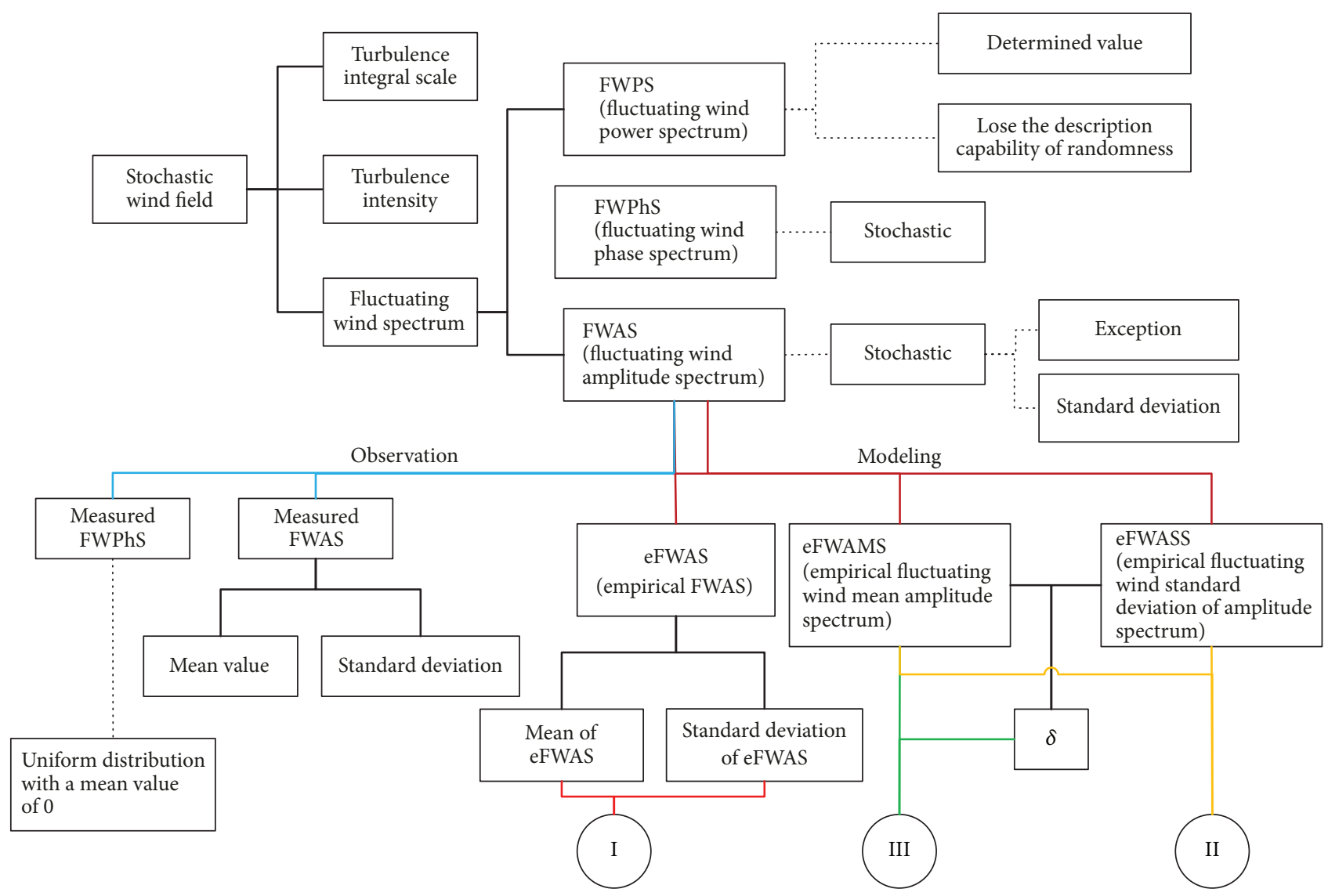

FIGURE 14: Empirical fluctuating wind model concept system. 


\section{Acknowledgments}

This work is supported by the National key R\&D Program of China (2017YFF0205605); Shanghai Urban Construction Design Research Institute Project "Bridge Safe Operation Big Data Acquisition Technology and Structure Monitoring System Research"; and the Ministry of Transport Construction Science and Technology Project "Medium-Small Span Bridge Structure Network Level Safety Monitoring and Evaluation.”

\section{References}

[1] A. G. Davenport, "How can we simplify and generalize wind loads?" Journal of Wind Engineering \& Industrial Aerodynamics, vol. 54-55, no. 94, pp. 657-669, 1995.

[2] S. Campbell, K. C. S. Kwok, P. A. Hitchcock, K. T. Tse, and H. Y. Leung, "Field measurements of natural periods of vibration and structural damping of wind-excited tall residential buildings," Wind and Structures, An International Journal, vol. 10, no. 5, pp. 401-420, 2007.

[3] B. Yu, A. Gan Chowdhury, and F. J. Masters, "Hurricane wind power spectra, cospectra, and integral length scales," Boundary Layer Meteorology, vol. 129, no. 3, pp. 411-430, 2008.

[4] H. Wang, T. Guo, T. Y. Tao, and A. Q. Li, "Study on wind characteristics of runyang suspension bridge based on long-term monitored data," International Journal of Structural Stability \& Dynamics, vol. 16, no. 4, Article ID 1640019, 2015.

[5] S. O. Rice, "Mathematical analysis of random noise," Bell Labs Technical Journal, vol. 24, pp. 46-156, 1945.

[6] V. Kármán and S. Theodore, "Progress in the statistical theory of turbulence," Proceedings of the National Academy of Sciences, vol. 34, no. 34, pp. 530-539, 1948.

[7] S. Emil, Wind Effects on Structures: Fundamentals and Applications to Design, 3rd edition, 1996.

[8] I. Goswami, R. H. Scanlan, and N. P. Jones, "Vortex-induced vibration of circular cylinders. I: experimental data," Journal of Engineering Mechanics, vol. 119, no. 11, pp. 2270-2287, 1993.

[9] M. Shinozuka, "Digital simulation of random processes and its applications," Journal of Sound and Vibration, vol. 25, no. 1, pp. 111-128, 1972.

[10] M. Shinozuka, "Simulation of multivariate and multidimensional random processes," The Journal of the Acoustical Society of America, vol. 49, no. 1, pp. 357-367, 1971.

[11] P. D. Spanos and M. P. Mignolet, "Simulation of homogeneous two-dimensional random fields: part ii-ma and arma models," Journal of Applied Mechanics, vol. 59, no. 2S, pp. S270-S277, 1992.

[12] J. Li and L. L. Zhang, "Research on the random fourier spectrum of observational wind," Journal of Vibration Engineering, vol. 20, no. 1, pp. 66-72, 2007 (Chinese).

[13] E. G. Larsson, J. Li, and P. Stoica, "High-Resolution Nonparametric Spectral Analysis: Theory and Applications," 2003.

[14] P. Stoica, H. Li, and J. Li, "New derivation of the APES filter," IEEE Signal Processing Letters, vol. 6, no. 8, pp. 205-206, 1999.

[15] J. Li and P. Stoica, "An adaptive filtering approach to spectral estimation and SAR imaging," IEEE Transactions on Signal Processing, vol. 44, no. 6, pp. 1469-1484, 1996.

[16] J. Capon, "Maximum-likelihood spectral estimation," in Nonlinear Methods of Spectral Analysis, vol. 34 of Topics in Applied Physics, pp. 155-179, Springer Berlin Heidelberg, Berlin, Heidelberg, 1983.
[17] P. Stoica, A. Jakobsson, and J. Li, "Matched-filter bank interpretation of some spectral estimators," Signal Processing, vol. 66, no. 1, pp. 45-59, 1998.

[18] "Wind-resistent design specification for highway bridges, JTG/T D60-01-2004, R.P. China”.

[19] X. Haifan et al., Modern Theory and Practice on Bridge Wind Resistance, China Communications Press, Beijing, China, 2005.

[20] H. U. Xiaohong, "The fitting of $2 \mathrm{~d}$ fluctuating wind spectrum for the actual measurement data of 'pba' typhoon in Shanghai," Structural Engineers, 2002 (Chinese).

[21] C. Meyer, M. Huglo, and N. C. Phillips, Canadian Structural Design Manual, 1970, supplement 4, The National Building Code of Canada, 1970. 


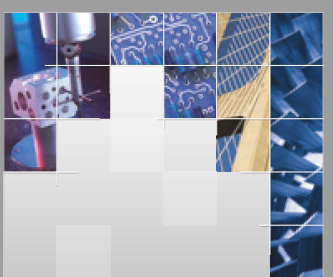

\section{Enfincering}
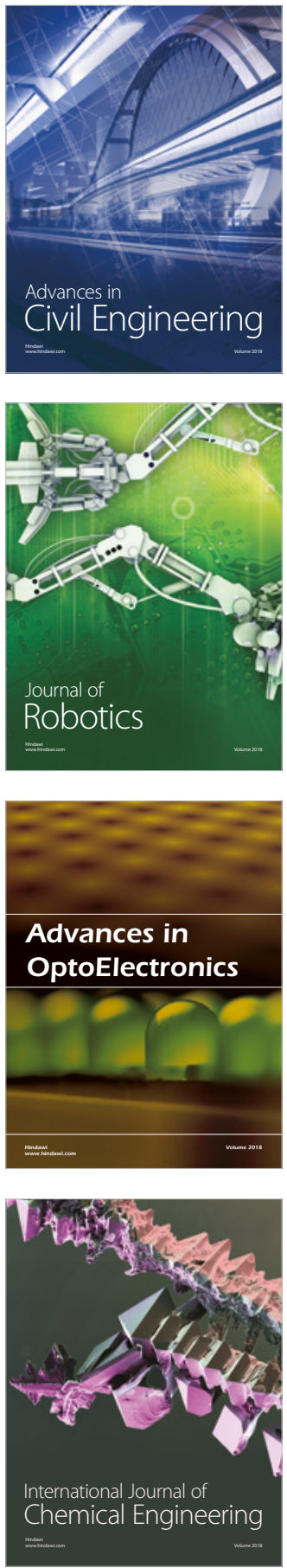

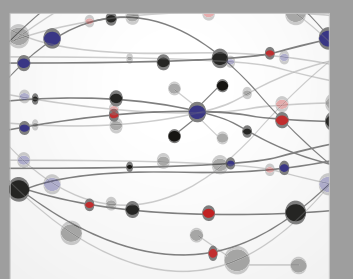

\section{Rotating \\ Machinery}

The Scientific World Journal

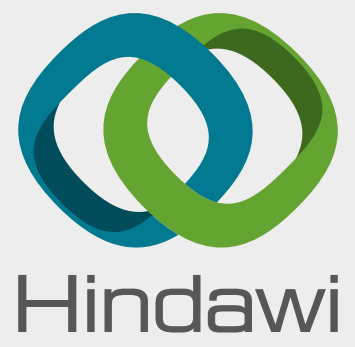

Submit your manuscripts at

www.hindawi.com
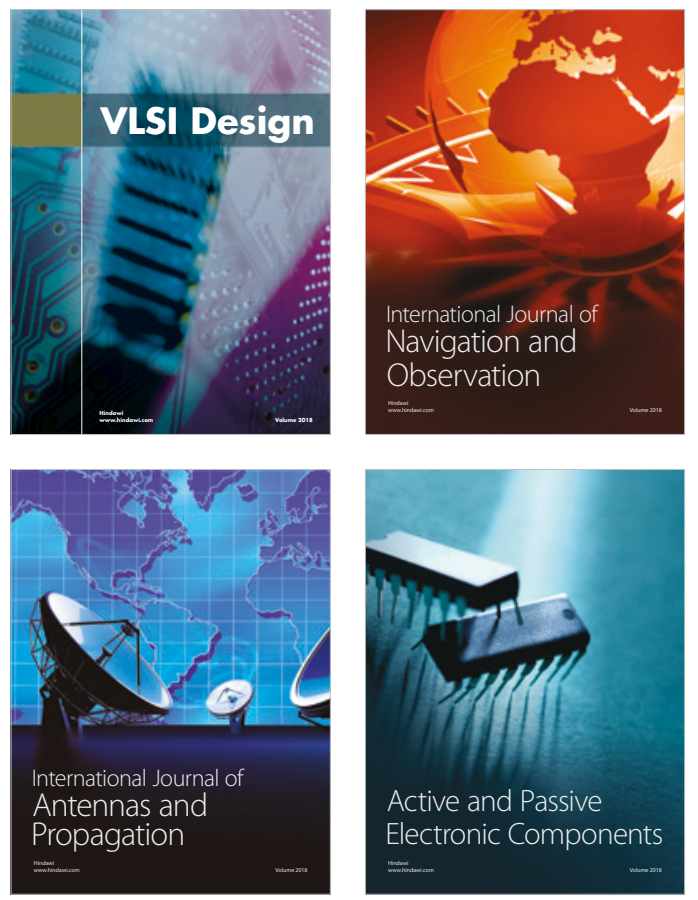
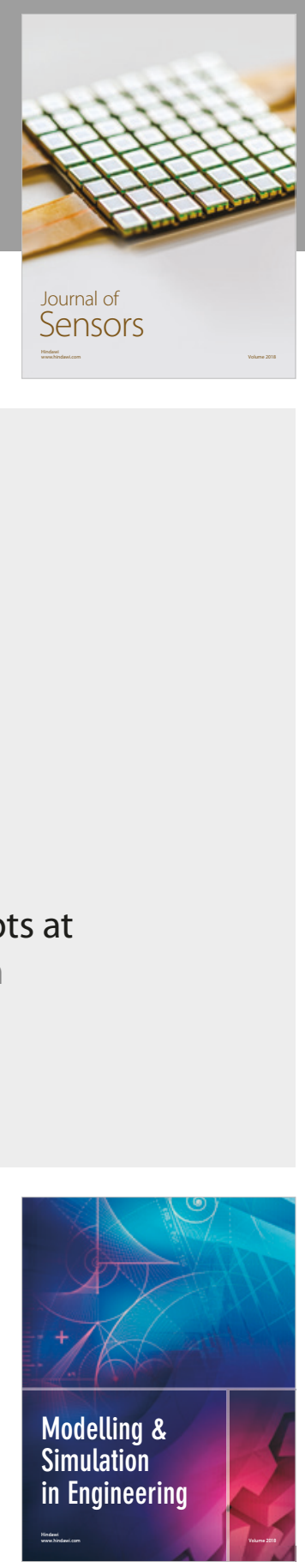

\section{Advances \\ Multimedia}
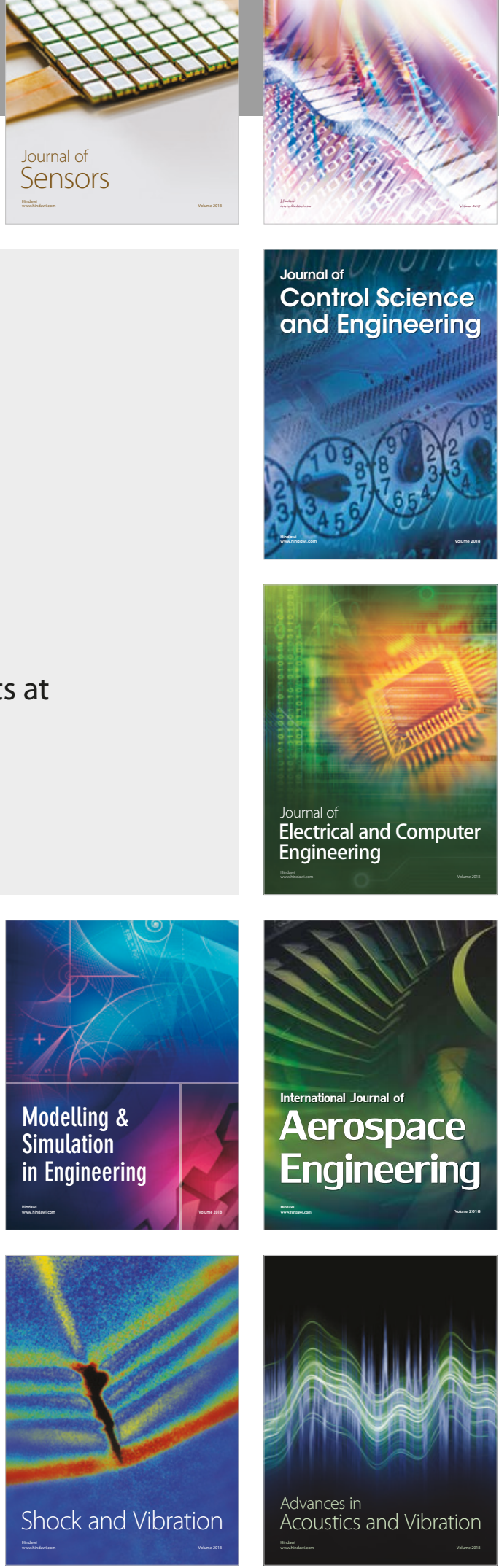